\title{
Exploring barriers and facilitators to integrated hypertension-HIV management in Ugandan HIV clinics using the Consolidated Framework for Implementation Research (CFIR)
}

Martin Muddu ( $\nabla$ muddu.martin@gmail.com )

Makerere University Joint AIDS Program (MJAP)

Andrew K. Tusubira

Uganda Initiative for Integrated Management of Non-Communicable Diseases

Brenda Nakirya

Uganda Initiative for Integrated Management of Non-Communicable Diseases

Rita Nalwoga

Uganda Initiative for Integrated Management of Non-Communicable Diseases

Fred C. Semitala

Department of Medicine, Makerere University College of Health Sciences, Kampala, Uganda

Ann R. Akiteng

Uganda Initiative for Integrated Management of Non-Communicable Diseases

Jeremy I Schwartz

Section of General Internal Medicine, Yale School of Medicine

Isaac Ssinabulya

Uganda Heart Institute

Research

Keywords: Barriers, Facilitators, Hypertension and HIV Integration, Uganda, CFIR

Posted Date: January 9th, 2020

DOI: https://doi.org/10.21203/rs.2.20444/v1

License: (c) (i) This work is licensed under a Creative Commons Attribution 4.0 International License.

Read Full License

Version of Record: A version of this preprint was published at Implementation Science Communications on May 4th, 2020. See the published version at https://doi.org/10.1186/s43058-020-00033-5. 


\section{Abstract}

\section{Background}

Persons Living with HIV (PLHIV) receiving antiretroviral therapy have increased risk of cardiovascular disease (CVD). Integration of services for hypertension (HTN), the primary CVD risk factor, into HIV clinics is recommended in Uganda. Our prior work demonstrated multiple gaps in implementation of integrated HTN care along the HIV treatment cascade. In this study, we sought to explore barriers to, and facilitators of, integrating HTN screening and treatment into HIV clinics in Eastern Uganda.

\section{Methods}

We conducted a qualitative study at three HIV clinics with low, intermediate, and high HTN care cascade performance, which we classified based on our prior work. Guided by the Consolidated Framework for Implementation Research (CFIR), we conducted semi-structured interviews with health services managers, health care providers and hypertensive PLHIV $(n=83)$. Interviews were transcribed verbatim. Three qualitative researchers used both deductive (CFIR model-driven) and inductive (open coding) methods to develop relevant codes and themes. Ratings were performed to determine valence and strengths of each CFIR construct regarding influencing HTN/HIV integration.

\section{Results}

Of the $39 \mathrm{CFIR}$ constructs assessed, 17 were relevant to either barriers or facilitators to HTN/HIV integration. Six constructs strongly distinguished performance and were barriers, three of which were in the Inner setting (Organizational Incentives \& Rewards, Available Resources, Access to Knowledge \& Information); two in Characteristics of individuals (Knowledge \& Beliefs about the Intervention and Selfefficacy) and one in Intervention characteristics (Design Quality \& Packaging). Four additional constructs were weakly distinguishing and negatively influenced HTN/HIV integration. There were four facilitators for HTN/HIV integration related to the intervention (Relative advantage, Adaptability, Complexity and Compatibility). The remaining three constructs negatively influenced HTN/HIV integration but were nondistinguishing.

\section{Conclusion}

Using the CFIR, we have shown that while there are modifiable barriers to HTN/HIV integration in the Inner setting, Outer setting, Characteristics of individuals and implementation Process, HTN/HIV integration is of interest to patients, health care providers and managers. Improving access to HTN care among PLHIV will require overcoming barriers and capitalizing on the facilitators using a health system strengthening approach. These findings are a springboard for designing contextually appropriate interventions for HTN/HIV integration in low- and middle-income countries.

\section{Background}


Persons living with HIV (PLHIV) and receiving antiretroviral therapy (ART) are at increased risk of cardiovascular disease (CVD) [1-3]. In Uganda, approximately $1 / 3$ of PLHIV aged $\geq 18$ years have hypertension (HTN), the leading cause of CVD and preventable mortality [4-12]. PLHIV with HTN have an increased risk of mortality compared to HIV negative persons [13].

The World Health Organization (WHO) and Uganda Ministry of Health (MoH) consolidated guidelines for HIV Care and Treatment recommend that all PLHIV should be screened for HTN at every visit to the HIV clinic. PLHIV who are diagnosed with HTN should receive treatment for both HIV and HTN as integrated services $[14,15]$. HTN/HIV integration provides patient-centred care compared with vertical programs and increases efficiency, by eliminating fragmentation and duplication of services [16]. However, there is little empiric evidence for HTN/HIV integrated services in Uganda.

HTN/HIV integration has been attempted in Uganda and Malawi by the SEARCH trial and the Lighthouse Trust program, respectively. However, although HTN screening was achieved for all patients in the HIV clinics under these programs, control of HTN among PLHIV who received antihypertensive medication remained suboptimal at about $30 \%[5,17]$.

We recently conducted a retrospective cohort study of 1649 PLHIV. We mapped the parallel care cascades for HTN and HIV within three high volume HIV clinics (average 3600 PLHIV) in Eastern Uganda and demonstrated suboptimal HTN screening, one year retention in HTN care, and HTN control of $27 \%, 57 \%$ and $24 \%$ respectively among PLHIV within a successful HIV program that has achieved the two of three UNAIDS 90-90-90 goals [18]. As a follow-up to that study, this qualitative study sought to determine barriers to, and facilitators of, integrating screening and treatment of HTN into HIV clinics in Eastern Uganda. Understanding the barriers and facilitators would inform the design of contextually appropriate implementation interventions for HTN/HIV integration in Uganda.

\section{Methods}

\section{Study design}

Using data from our previous retrospective cohort study, we graded HTN care cascade performance among the three HIV clinics as high, intermediate and low according to achievement in screening, diagnosis, initiation of treatment, retention, monitoring and control.

We utilized the Consolidated Framework for Implementation Research (CFIR) to explore barriers to, and facilitators of, HTN/HIV integration [18]. CFIR provides a pragmatic structure for identifying potential influences on implementation of interventions in health systems at multiple levels [19, 20]. CFIR organizes conceptual elements across theories and disciplines into 39 constructs which are then organized in five key domains. All constructs interact to affect the process and effectiveness of implementation [21]. CFIR's five major domains include: Intervention characteristics, Outer setting, Inner setting, Characteristics of individuals, and Implementation Process [22]. (Figure 1). Barriers to, and 
facilitators of, HTN/HIV integration (Intervention) were compared across the three HIV clinics to understand the strength of their influence on HTN screening and treatment among PLHIV.

\section{Study setting}

We conducted this study at three HIV clinics (The AIDS Support Organization (TASO) Tororo, Nagongera Health Centre IV and Mulanda Health Center IV) and the District Health Office of Tororo District, Eastern Uganda.

The Ugandan public-sector healthcare system is hierarchical in nature and comprises the national (Ministry of Health [MoH]), sub-national (Regional), district, health facility and community levels. $\mathrm{MoH}$ develops guidelines for health services and rolls them out through the regions to the districts. The District Health Team (DHT) leads and coordinates guideline implementation at health facilities.

HIV clinics are the designated treatment centers for HIV, HIV-associated opportunistic infections and other HIV-associated co-morbidities. They are physically situated as outpatient departments within health centers and hospitals. We selected these three HIV clinics because they are the largest in Tororo district, providing care to approximately 7500, 1400, and 1100 PLHIV, respectively. They are housed within public health facilities with support from both the Government of Uganda and the President's Emergency Fund for AIDS Relief (PEPFAR). The clinics are staffed by various cadres of health workers including clinicians, nurses, midwives, and HIV peer counsellors. Each HIV clinic offers a full spectrum of HIV services including screening, ART, viral load testing, as well as screening for and treating opportunistic infections. Each also has the mandate to screen for, and manage NCDs such as HTN and diabetes. Based on existing processes in place at the time of data collection, within a given clinical encounter, BP is measured by the clinician at his/her discretion [18]. If a patient is diagnosed with HTN (by measurement or previous history), the clinician typically prescribes both ART and antihypertensive medicine simultaneously and the client is given one follow-up appointment for both conditions. All medicines at facility pharmacies are obtained from the centralized National Medical Stores (NMS). PEPFAR provides funds to NMS to procure medicines specifically for HIV and opportunistic infections. Medicines for HTN and other NCDs are procured on request by the health facilities via general funds allocated to each health facility by $\mathrm{MoH}$. If medicines are out of stock at the facility pharmacy, the patient is advised to purchase them from a private sector pharmacy of the patient's choice.

Clinic providers are routinely oriented to national HIV treatment guidelines which recommend screening for NCDs and their risk factors. In addition, clinical support discussions about challenging HIV/NCD cases were also used to build capacity for NCD/HIV integration among clinicians.

\section{Study participants and Sampling}

We interviewed purposively selected healthcare providers and patients living with both HIV and HTN. Eligible healthcare care providers were individuals who had responsibility to treat patients in the HIV clinics or leadership roles at the respective health facilities or at the district health office. These health 
care providers were knowledgeable about and actively involved in HIV service delivery at their clinics or at the district health office. Eligible patients were PLHIV with hypertension attending one of the three HIV clinics. Patients with a mental disability were excluded. We approached patients through telephone calls and healthcare providers face to face. All participants approached accepted to participate. We recruited participants until we achieved data saturation.

\section{Data collection}

We used semi-structured interview guides developed based on the five domains of CFIR [19]. The interviews had open ended questions reflecting patient, provider and health care managers' perspectives and perceptions about HTN/HIV integration. Prior to data collection, we pretested the interview guides with health care providers and hypertensive PLHIV at TASO Tororo who were not participating in the study. AKT, DBN and RN conducted the FGDs and IDIs. AKT is male public health specialist while DBN and $\mathrm{RN}$ are female social scientists with expertise in public health. All three authors who conducted interviews were not part of the healthcare team at the HIV clinics but had experience in conducting FGDs and IDIs. We conducted six FGDs (two per HIV clinic) with patients in each group consisting of ten hypertensive PLHIV lasting 60 minutes. We conducted twelve IDIs with hypertensive PLHIV, four at each HIV clinic and eleven KIls each lasting 30 minutes. We used patient IDIs to obtain individual lived experiences, while FGDs explored shared experiences among hypertensive PLHIV. The FGDs and interviews were conducted in a private space within the HIV clinics. All interviews were audio-recorded and transcribed verbatim.

\section{Data analysis}

\section{Qualitative data coding}

After transcribing, a research team with expertise in social sciences, public health and clinical care was established comprising three members (AKT, DBN, RN), who conducted thematic content analysis. The team coded transcripts using both deductive (guided by CFIR as a coding framework) and inductive (through open coding to obtain new themes that may arise) approaches. The coding process was guided by the consensual qualitative research (CQR) procedure [23].

First, each research team member read three transcripts independently and identified preliminary codes. Through a series of meetings, discussing coding differences, an initial codebook was agreed upon. To organize and manage the large amount of data, all transcripts were then coded utilizing Atlas.ti (version 7) software while applying the codebook and giving an allowance for new codes. An external researcher independently coded six of the transcripts to establish inter coder reliability (Kappa 0.80). A final codebook and subthemes were resolved by the researchers through more meetings and these were mapped to the CFIR domains and constructs (Table 3).

\section{Defining unit of analysis and performance criteria}


The three clinics were our units of analysis. We used data from our recent retrospective cohort study [18] which assessed performance of each HIV clinic across HTN care cascade steps including: Screening, Diagnosis, Initiation of Treatment, Retention into care, Monitoring and BP Control (This assessment is also highlighted in figure 2). Generally, the most significant care gaps were identified in Screening and BP control[18]. Basing on the cascade performance reported, we classified the three HIV clinics as high, intermediate, or low performing. (Figure 2).

\section{Rating the CFIR constructs and interpretation}

Ratings were performed to determine valence, which assesses whether the construct had a positive, neutral, or negative influence on implementation of integrated HTN/HIV care, and strength which is the degree of its influence. The coded text was then subjected to a rating process based on criteria shown in Table 1 [24]. We used a consensus process to assign a rating to each construct obtained from each site based on the coded text. We based the rating of constructs on the level of agreement among study participants interviewed, strength of language, and use of concrete examples to emphasize responses (Table 1). Positive influence indicated a facilitator of HTN/HIV integration while negative influence indicated a barrier. After rating all constructs obtained from the three HIV clinics, we developed a matrix that listed the ratings for the CFIR construct for each of the clinics. We then focused our analysis on discerning patterns across the three HIV clinics. Using relative rating, but not absolute figures, we compared ratings across the HIV clinics and determined which constructs distinguished performance, either strongly or weakly, or did not distinguish but influenced performance either negatively or positively. Complexity was 'reverse rated' to be consistent with the other constructs; i.e., a positive sign denotes perception of simplicity and a negative sign denotes complexity in implementation [22, 24, 25]. (Table 3). We extracted specific quotations from the transcripts illustrating verbatim expressions of matters that appeared important. We followed the consolidated criteria for reporting qualitative research (COREQ) checklist in developing the manuscript [26]. We presented the findings to healthcare providers and patient representatives at the participating HIV clinics and the district.

\section{Results}

TASO Tororo was the highest performing for all the six cascade steps. Mulanda HC IV was intermediate for screening, diagnosis, initiation of treatment, and control but lowest for retention and monitoring. Nagongera HC IV was the lowest for all the six cascade steps having achieved the same with Mulanda $\mathrm{HC}$ IV in retention and monitoring. (Figure 2).

Apart from the DHT member, the male to female ratios of participants were 1:1 in all FGDs, IDIs and KIls. (Table 2).

Of the 39 CFIR constructs assessed, 17 were relevant to either barriers or facilitators to HTN/HIV integration. We found six constructs which strongly distinguished performance, three of which were in the Inner setting (Organizational Incentives \& Rewards, Available Resources, and Access to Knowledge \& Information); two in Characteristics of individuals (Knowledge \& Beliefs about the Intervention and Self- 
efficacy) and one in Intervention characteristics (Design Quality \& Packaging). Four additional constructs distinguished performance weakly. All ten distinguishing constructs negatively influenced HTN/HIV integration. There were four constructs [Relative advantage, Adaptability, the simplicity (non-complex nature) of the intervention and Compatibility] which had positive influence for HTN/HIV integration at all the three HIV clinics but did not distinguish performance. These four factors related to the intervention are the key facilitators for HTN/HIV integration. The remaining three constructs negatively influenced HTN/HIV integration but were non- distinguishing. (Table 3). Below, we present the detailed results in the context of the five CFIR domains.

\section{Intervention Characteristics}

Healthcare providers at all three HIV clinics perceived HTN/HIV integration as a relative advantage and more effective compared to alternative modes of care for hypertensive PLHIV. Health care providers noted that integrated HTN/HIV saves time since patients received care for both HTN and HIV in the same clinic on the same appointment date, hence reducing costs on transport and improving retention.

"It is extremely important because if I were a patient having two chronic conditions, I would not want to spend my time going to a hospital for condition $A$ and then go to another for condition B. HIV clinics should be a one-stop centre ... (KII, health facility manager HIV clinic $\mathrm{C}$ )

Adaptability was a facilitator for HTN/HIV integration since respondents at all the clinics perceived that HTN/HIV integration fits within their routine care provision. Health care providers stated: "HTN services can be tailored and refined to meet health needs of PLHIV."

"Yes, hypertension management fits very well within our HIV care programs. (KII, health facility manager HIV clinic C).

Inadequacies in the design quality and packaging of HTN/HIV integration especially when the program was being initiated was a barrier to the implementation. Health care providers reported that the implementation of HTN/HIV integration policy by MoH was suboptimal. This strongly distinguishing construct mainly affected the low performing clinics. Health care providers at these clinics reported insufficiencies in the preparation, packaging and support for HTN/HIV integration including lack of: staff and professional training systems such as initial orientation to the new health guidelines: These healthcare providers noted that;

"The introduction of this program was not well communicated to the staff. A clear plan for HTN service provision should have been given to us and may be an official launch involving patients and we healthcare providers. (KII, facility manager, HIV clinic A).

Although the presentation and design of the integration were cited as insufficient, healthcare providers at the high implementing HIV clinic noted that they receive some support through their organisation arrangements which has helped them to get acquainted with the HTN/HIV integration program. 
“We didn't have any capacity building at the start. However, as an organisation, we regularly have Continuous Medical Education (CME) on non-communicable diseases to boost the staffs' knowledge. So we got some information on the suggested HTN/HIV integration strategies." (KII, clinician, site C).

Complexity was a facilitator for HTN/HIV integration. Across the HIV clinics, healthcare providers perceived provision of HTN care services as a task which was not complex and that activities for integrated HTN/HIV care were straightforward. However, an increased number of hypertensive PLHIV at the HIV clinics with few healthcare providers would increase provider work load and complexity of handling all patients. A member of the DHT stated:

"For now, running the HIV clinics when providing care for opportunistic infections (Ols) and other NCDs isn't difficult at these clinics. Although .... when patients are many, it could be challenging for the few staff to offer care for the many tasks."(KII, DHT).

\section{Outer Setting}

Patient needs and resources was a barrier to HTN/HIV integration and weakly distinguished performance across the three HIV clinics. Both health care providers and patients were in agreement that, to a large extent, HTN services at the HIV clinics were suboptimal to meeting the needs of PLHIV. Providers at lower performing HIV clinics added that HTN services were not adequately prioritized as evidenced by the low demand.

"Save for the good HIV care, it [HTN management] is almost not provided at this centre. ... So, I seek treatment outside this centre." (IDI 1, HIV clinic A).

"Patients are mainly interested in getting the HIV medicine [ART] refills. They do not demand for extra care unless their health has deteriorated. Most are not aware of other clinical service we provide including HTN management." (Lead nurse HIV clinic B).

"We also like to work on all patients' conditions but we are sometimes limited by resources. Even now, not much is being done to support HTN integration." (KII, DHO).

Because the low implementing HIV clinics were not in a position to meet PLHIV's needs for HTN management, hypertensive PLHIV were often referred to other health facilities, a strategy which HTN patients were not comfortable with.

"I was sent to Tororo general clinic (private for profit) to get checked and I was diagnosed with hypertension. I have the results with me but I'm always told to go back for care at the hospital." (P2, FGD 2, HIV clinic A).

External Policy and Incentives was a weekly distinguishing construct that negatively influenced HTN/HIV integration. There were few external strategies for HTN/HIV integration through policies and guidelines. 
Although at the high performing site, healthcare providers stated implementing the national HIV guidelines, their emphasis was on the HIV component:

Currently we are implementing the 2016 National guidelines for HIV/AIDS with emphasis on test and treat." (Lead clinician HIV clinic C).

This construct was highlighted by healthcare providers at the low performing site that they lacked comprehensive guidelines for HTN/HIV integration:

"We also lack specific standard operating procedures or documents to be followed in the HTN/HIV integration." (KII, Lead clinician HIV clinic A).

Besides, healthcare providers also reported insufficient support supervision from the health authorities including $\mathrm{MoH}, \mathrm{DHT}$, and implementing partners in relation to HTN management.

"No! We have not received any supervision at the HIV clinic, may be at the OPD [outpatient department]. Besides, when we are asked about the clinic, we are often asked about HIV care and supplies". (KII, Lead nurse HIV clinic A).

\section{Inner setting}

Three sub-constructs under Implementation Climate were relevant to HTN/HIV integration. These were: compatibility, relative priority and organization incentives and rewards. Compatibility was a facilitator for HTN/HIV integration. Healthcare providers perceived that HTN/HIV integration was compatible and would fit within the existing workflows at the HIV clinics. One of the healthcare providers noted:

"Yes, hypertension services do fit within our routine HIV care service provision." (KII, lead clinician HIV clinic A).

Relative priority, was a weakly distinguishing construct which negatively influenced HTN/HIV integration. Although health care providers reported that HTN management would fit within the HIV clinic workflow, we found a relatively lower priority attached to HTN management by the healthcare providers compared to HIV care. Healthcare providers reported irregular provision of HTN services at the HIV clinic and providers from the low and intermediate performing facilities explicitly stated that:

"Basically, we provide the necessary HIV care services... and often patients are many; much work to do, so we prioritize HIV care". (KII, lead clinician HIV clinic B).

"We often check the BP for patients with known hypertension and prescribe for them the medicines. However, those ones who are not yet known, we may check once in six months or when they complain with signs and symptoms suggestive of hypertension." (KII, Lead clinician HIV clinic A).

Organization incentives and rewards was a barrier that strongly distinguished HTN/HIV integration. While healthcare providers at the high performing HIV clinic seemed to be motivated by the availability of 
equipment and other supplies to manage HTN, providers at the lower preforming sites expressed the need to receive incentives like functional BP equipment and HTN treatment supplies or rewards to motivate their action for additional HTN services:

"Even the medicines and other equipment should be available. But we are still struggling to get better BP machines." (KII, Lead clinician HIV clinic A).

"The HIV clinic does not receive special facilitation [payment] for managing hypertension cases." (KII, Lead clinician HIV clinic B).

The two sub-constructs Available resources and Access to knowledge and information under the Readiness for implementation construct were barriers to HTN/HIV integration.

Available resources for HTN services within the HIV clinics was a strongly distinguishing factor because the high performing site reported having adequate resources including staff who manage HTN, functional BP machines at each department, and medicines for HTN:

"Yes, I think we have adequate support. We have resources like drugs, equipment they are there, human resource." (KII, Lead clinician HIV clinic C).

On the contrary, low and intermediate performing HIV clinics reported lack of enough equipment especially functional BP machines. They also experience frequent stockouts of the medicines for HTN and the lack of specific funding towards HTN services:

"... sometimes the $[\mathrm{BP}]$ machine at the clinic breaks down and it takes a longer process to get another. Yes. Most times we experience stockout of these HTN drugs, so we end up referring the patients." (KII, facility manager HIV clinic B).

Similarly, access to information and knowledge was a barrier that strongly distinguished HTN/HIV integration between high and low performing clinics. At the high performing clinic, healthcare providers reported often having trainings including continuing medical education (CMEs) sessions. Some of the CMEs would be refresher trainings and education on NCDs care including HTN.

"We always have CMEs on hypertension cases, we have had workshops and trainings, management of Ols and hypertension is part of it, that one has been done." (KII, Lead clinician HIV clinic C).

On the other hand, healthcare providers at lower performing sites stated low access to information and knowledge about HTN care for PLHIV. They identified lack of trainings, few available trained staff at their sites, and poor learning environment as contributing factors.

"We have not had [trainings or capacity building sessions on hypertension management] for some time and that is the challenge that we have." (KII, facility manager A).

\section{Characteristics of individuals}


Knowledge and beliefs about HTN/HIV integration was a strongly distinguishing construct and a barrier. At the high and intermediate sites, healthcare providers were acquainted with knowledge and skill to offer HTN services like BP measurement and prescription of medicine while at the low performance site, some of the health care providers lacked the skills to appropriately screen and treat HTN:

"I realized that health workers would report inconsistent BP measurements from patients. In case the triage says the blood pressure is high, I would measure again in the clinical room. Okay, there are some acceptable variations but there are those that are out of range. So it creates the need for more trainings." (KII, lead clinician HIV clinic A).

Self-efficacy, a strongly distinguishing construct, was a barrier to HTN/HIV integration at the low and intermediate sites. While healthcare providers at the high performing site expressed confidence in their own ability to screen and treat HTN, some providers at the lower performing clinics expressed low selfefficacy to screen and prescribe medicines for HTN. One health care provider stated:

"Patients often present to us [with] different symptoms, in case you follow only these symptoms, you may think its pressure yet it's not. I have sometimes used the BP machine, but because I don't use it frequently, I don't think I get exact measurements to conclude that one has pressure." (KII Lead nurse HIV clinic B).

\section{Implementation process}

Execution of HTN/HIN integration was a weakly distinguishing construct as responses across the three clinics expressed sub optimal HTN services. A healthcare providers at the low performing HIV clinic mentioned:

"... we concentrate on the HIV care package... We sometimes include hypertension, but not frequently. (KII, lead clinician HIV clinic A).

Results for the three CFIR constructs which were non-distinguishing and negatively influencing HTN/HIV integration namely: Peer pressure, Reflecting and Evaluating and Engaging opinion leaders are presented in the appendix.

\section{Discussion}

This study sought to evaluate factors that influence the integration of screening and treatment of HTN into the HIV program in Uganda, using the CFIR. We used valance rating to identify factors which distinguished performance for integrated HTN/HIV between the high and low performing HIV clinics. We found ten CFIR constructs which distinguished performance, four of which were in the inner setting domain. Six of the constructs distinguished performance strongly while the remaining four weakly. All ten distinguishing constructs negatively influenced HTN/HIV integration at the low and intermediate performing HIV clinics as compared to the high performing clinic. 
There were four constructs which positively influenced HTN/HIV integration at all the three HIV clinics but did not distinguish performance. These included: Relative advantage, Adaptability, Complexity of the intervention, and Compatibility of HTN care with existing HIV services. We view these as the key facilitators for integrated HTN/HIV services in our setting. In agreement with the facilitators we identified for integrated HTN/HIV services, there is increasing demand for integrated rather than vertically oriented HTN and HIV services in low-and middle income countries (LMIC) [5, 16, 17, 27-29]. Although some studies have assessed barriers and facilitators for HTN care in the HIV program, this is the first study in Africa to assess factors that influence HTN/HIV integration using the CFIR.

Adaptability was a positive influencer of integrated HTN/HIV services. Health care providers and leaders, especially in the high performing clinic, perceive that integrated HTN/HIV care can be adapted and tailored to fit the workflow of the HIV clinics to meet patient needs. HIV services were originally established as vertically oriented entities to address the emergency nature of HIV. Now that HIV has evolved into a chronic disease, largely due to these intensive, vertical efforts, leveraging successful HIV programs for NCD integration is highly recommended [16, 29]. A large clinical trial in Uganda that integrated HTN care into the HIV program showed that HTN control is better achieved in the HIV program as compared to the non-HIV population [30]. This is a true demonstration of the adaptability of integrated HTN/HIV service to the HIV program [30].

Health care providers noted that HTN/HIV integration was compatible with the existing services in the HIV clinics and workflows. A project in Malawi that integrated screening and treatment of HTN into the HIV clinics demonstrated that HTN care was compatible with HIV services [5]. Indeed, as we demonstrated previously. the care cascades for both conditions can be perfectly aligned [18].

All ten constructs which distinguished performance had a negative influence upon HTN/HIV integration. Design quality and packaging was a barrier to HTN/HIV integration. Health care providers from the lower performing clinics were not oriented on HTN/HIV integration, unlike those at the high performing site. Additionally, Ugandan HIV programs will need to adopt the WHO target of $50 \%$ for HTN screening, treatment, retention in care and control. Achieving this will require quality improvement efforts and integration of CVD indicators into routinely collected data at National, regional, district and health facilities [27].

\section{Conclusions}

Using the CFIR framework, we have shown that there are modifiable barriers to integrating HTN services into the HIV clinic in the inner setting, outer setting, characteristics of individuals and implementation process. Integrated HTN and HIV care is of great interest to both patients and health care providers. Improving access to HTN and other CVD care among PLHIV will require overcoming these barriers and capitalizing on the facilitators identified using a health system strengthening approach [5]. Findings from this study provide a springboard for designing contextually appropriate multicomponent interventions for HTN/HIV integration in Uganda and other LMICs. To further build the case for integrated HTN/HIV 
services, future research should determine the cost, cost effectiveness and treatment outcomes for both HTN and HIV from integrated HTN/HIV services [16, 27].

\section{Abbreviations}

CFIR Consolidated Framework for Implementation Research

HTN Hypertension

PLHIV Persons Living with HIV

CVD Cardiovascular Disease

ART Antiretroviral Therapy

WHO World Health Organization

$\mathrm{MoH} \quad$ Ministry of Health.

FGD Focus group discussion

KII Key Informant Interview

IDI In-depth interview

DHO District Health Officer

DHT District Health Team

LMICs Low-and Middle Income Countries

\section{Declarations}

\section{Ethics approval and consent to participate}

This study was approved by the TASO Institutional Review Board (IRB) number TASOREC/65/17-UG-REC009 and the Uganda National Council for Science and Technology (UNCST) number SS4583. Permission (written consent) to access the HIV clinics and medical records was sought from the DHO and health facility in-charges respectively. Written informed consent was sought from each participant after explaining the purpose of the study, benefits, approximate time of interview and an assurance of confidentiality of the study results. Selected individuals were asked if they would like to participate, and if the response was affirmative, the interviewer would give them a consent form to be signed as evidence of acceptance to take part in the study. In case a participant was not willing to be audio recorded for key informant or in-depth interviews, interview notes were taken. Privacy was maintained during data collection, analysis and storage. All identifiable information was removed from final records after data 
collection to ensure participant anonymity and only the core research team and Pls had access to the deidentified data.

\section{Consent for publication}

Not applicable

\section{Availability of data and materials}

The datasets used and/or analysed during the current study are available from the corresponding author on reasonable request.

\section{Competing interests}

The authors declare that they have no competing interests

\section{Funding}

Research reported in this manuscript was supported by the Fogarty International Center and the National Heart, Lung, and Blood Institute (NHLBI) at the National Institutes of Health (NIH) under the Global Health Equity Scholars Consortium at Yale University (D43TW010540). Dr. Martin Muddu was also supported by the Fogarty International Center of the NIH under Award Number D43 TW010037. Dr. Ssinabulya Isaac was supported by the Nurture fellowship Grant Number D43TW010132. The content is solely the responsibility of the authors and does not necessarily represent the official views of the NIH. The funder had no role in the study design, data collection, analysis or interpretation. Martin Muddu had full access to all the data and had the final responsibility for the decision to submit the manuscript for publication.

\section{Author contributions}

MM, IS, JIS and AKT were responsible for the design of the study and interpretation of data. MM, AKT, BN and RN led data collection and interpretation. AKT, BN and RN performed data analysis. ARA participated in study design and data interpretation. FCS participated in the interpretation of data. All authors participated in writing the initial draft of the manuscript. Martin Muddu and Jeremy and Isaac Ssinabulya participated in writing the final manuscript. All authors read and approved the final manuscript before submission.

\section{Acknowledgement}

We are grateful to the following persons for their invaluable support: The District Health Officer and the District Health Team of Tororo District, the staff of TASO Tororo, Nagongera HC IV and Mulanda HC IV HIV clinics and all the research assistants who participated in data collection for this study.

\section{References}


1. Haldane V, Legido-Quigley H, Chuah FLH, Sigfrid L, Murphy G, Ong SE et al. Integrating cardiovascular diseases, hypertension, and diabetes with HIV services: a systematic review. AIDS Care. 2018 Jan; 30(1):103-115.

2. Magafu MG, Moji K, Igumbor EU, Magafu NS, Mwandri M, Mwita JC et al. Non-communicable diseases in antiretroviral therapy recipients in Kagera Tanzania: a cross-sectional study. Pan Afr Med J. 2013 Nov 6; 16:84.

3. Fahme SA, Bloomfield GS, Peck R. Hypertension in HIV-infected adults: novel pathophysiologic mechanisms. Hypertension. 2018 Jul; 72(1):44-55.

4. Gakidou E, Afshin A, Abajobir A. A, Abate K.H, Abbafati C, Abbas K.M et al. Global, regional, and national comparative risk assessment of 84 behavioural, environmental and occupational, and metabolic risks or clusters of risks, 1990-2016: a systematic analysis for the Global Burden of Disease Study 2016. Lancet. 2017 Sep 16;390(10100):1345-1422.

5. Patel P, Speight C, Maida A, Loustalot F, Giles D, Phiri S et al. Integrating HIV and hypertension management in low-resource settings: Lessons from Malawi. PLoS Med. 2018 Mar 7; 15(3):e1002523.

6. Lunyera J, Kirenga B, Stanifer JW, Kasozi S, van der Molen T, Katagira Wet al. Geographic differences in the prevalence of hypertension in Uganda: Results of a national epidemiological study. PLoS One. 2018 Aug 1; 13(8):e0201001.

7. Rabkin M, Palma A, McNairy ML, Gachuhi AB, Simelane S, Nuwagaba-Biribonwoha $\mathrm{H}$ et al. Integrating cardiovascular disease risk factor screening into HIV services in Swaziland: lessons from an implementation science study. AIDS. 2018 Jul 1; 32 Suppl 1:S43-S46.

8. Patel P, Rose CE, Collins PY, Nuche-Berenguer B, Sahasrabuddhe VV, Peprah E et al.

Noncommunicable diseases among HIV-infected persons in low-income and middle-income countries: a systematic review and meta-analysis. AIDS. 2018 Jul 1; 32 Suppl 1:S5-S20.

9. Kwarisiima D, Balzer L, Heller D, Kotwani P, Chamie G, Clark T et al. Population-based assessment of hypertension epidemiology and risk factors among HIV-positive and general populations in rural Uganda. PLoS One. 2016 May 27; 11(5):e0156309.

10. Kazooba P, Kasamba I, Mayanja BN, Lutaakome J, Namakoola I, Salome T et al. Cardiometabolic risk among HIV-POSITIVE Ugandan adults: prevalence, predictors and effect of long-term antiretroviral therapy. Pan Afr Med J. 2017 May 15; 27:40.

11. Mateen FJ, Kanters S, Kalyesubula R, Mukasa B, Kawuma E, Kengne APet al. Hypertension prevalence and Framingham risk score stratification in a large HIV-positive cohort in Uganda. $J$ Hypertens. 2013 Jul; 31(7):1372-8; discussion 1378. 
12. Kalyesubula R, Kayongo A, Semitala FC, Muhanguzi A, Katantazi N, Ayers D et al. Trends and level of control of hypertension among adults attending an ambulatory HIV clinic in Kampala, Uganda: a retrospective study. BMJ Glob Health. 2016 Nov 24; 1(3):e000055.

13. Walsh KF, Lee MH, Martelly S, Pierre MM, Joseph J, Gustin M et al. Integrating hypertension services at an HIV clinic in Port-au-Prince, Haiti: A report from the field. J Clin Hypertens (Greenwich). 2018 Oct; 20(10):1485-1492.

14. World Health Organization (WHO). March 2014 supplement to the 2013 consolidated guidelines on the use of antiretroviral drugs for treating and preventing HIV infection: recommendations for a public health approach. Available at: http://www.who.int/ hiv/pub/guidelines/arv2013 /arvs2013upplement_march2014/en/ (accessed October 06, 2018)

15. Uganda Ministry of Health. Consolidated Guidelines for Prevention and Treatment of HIV in Uganda. 2016. Available at: http://library.health.go.ug /publications/service-delivery-diseases-controlprevention-communicable-diseases/hivaids/consolidated (accessed September 30, 2018)

16. Garrib A, Birungi J, Lesikari S, Namakoola I, Njim T, Cuevas L et al. Integrated care for human immunodeficiency virus, diabetes and hypertension in Africa. Trans R Soc Trop Med Hyg. 2018 Sep 28.

17. Kwarisiima D, Atukunda M, Owaraganise A, Chamie G, Clark T, Kabami J et al. Hypertension control in integrated HIV and chronic disease clinics in Uganda in the SEARCH study. BMC Public Health. 2019 May 6; 19(1):511.

18. Muddu M, Tusubira AK, Sharma SK, Akiteng AR, Ssinabulya I, Schwartz JI. Integrated Hypertension and HIV Care Cascades in an HIV Treatment Program in Eastern Uganda: a retrospective cohort study. J Acquir Immune Defic Syndr. 2019 Aug 15;81(5):552-561.

19. Damschroder LJ, Aron DC, Keith RE, Kirsh SR, Alexander JA, Lowery JC. Fostering implementation of health services research findings into practice: a consolidated framework for advancing implementation science. Implement Sci. 2009 Aug 7; 4:50.

20. Varsi C, Ekstedt M, Gammon D, Ruland CM. Using the Consolidated Framework for Implementation Research to Identify Barriers and Facilitators for the Implementation of an Internet-Based PatientProvider Communication Service in Five Settings: A Qualitative Study. J Med Internet Res. 2015 Nov $18 ; 17(11): \mathrm{e} 262$.

21. Kirk MA, Kelley C, Yankey N, Birken SA, Abadie B, Damschroder L. A systematic review of the use of the Consolidated Framework for Implementation Research. Implement Sci. 2016 May 17; 11: 72.

22. VanDevanter N, Kumar P, Nguyen N, Nguyen L, Nguyen T, Stillman F et al. Application of the Consolidated Framework for Implementation Research to assess factors that may influence 
implementation of tobacco use treatment guidelines in the Viet Nam public health care delivery system. Implement Sci. 2017 Feb 28; 12(1):27.

23. Depner RM, Grant PC, Byrwa DJ, Breier JM, Lodi-Smith J, Kerr CW et al. A consensual qualitative research analysis of the experience of inmate hospice caregivers: Posttraumatic growth while incarcerated. Death Stud. 2017 Apr; 41(4):199-210.

24. Damschroder LJ, Lowery JC. Evaluation of a large-scale weight management program using the consolidated framework for implementation research (CFIR). Implement Sci. 2013 May 10; 8:51.

25. Garbutt JM, Dodd S, Walling E, Lee AA, Kulka K, Lobb R et al. Barriers and facilitators to HPV vaccination in primary care practices: a mixed methods study using the Consolidated Framework for Implementation Research. BMC Fam Pract. 2018 May 7; 19(1):53.

26. Tong A, Sainsbury P, Craig J. Consolidated criteria for reporting qualitative research (COREQ): a 32item checklist for interviews and focus groups. Int J Qual Health Care. 2007 Dec; 19(6):349-57.

27. Osetinsky B, Hontelez JAC, Lurie MN, McGarvey ST, Bloomfield GS, Pastakia SD et al. Epidemiological And Health Systems Implications Of Evolving HIV And Hypertension In South Africa And Kenya. Health Aff (Millwood). 2019 Jul; 38(7):1173-1181.

28. Iwelunmor J, Ezechi O, Obiezu-Umeh C, Gbajabiamila T, Musa AZ, Oladele D at al. Capabilities, opportunities and motivations for integrating evidence-based strategy for hypertension control into HIV clinics in Southwest Nigeria. PLoS One. 2019 Jun 6; 14(6):e0217703.

29. Mutemwa M, Peer N, de Villiers A, Mukasa B, Matsha TE, Mills EJ et al. Prevalence, detection, treatment, and control of hypertension in human immunodeficiency virus (HIV)-infected patients attending HIV clinics in the Western Cape Province, South Africa. Medicine (Baltimore). 2018 Aug; 97(35):e12121.

30. Havlir DV, Balzer LB, Charlebois ED, Clark TD, Kwarisiima D, Ayieko J et al. HIV testing and treatment with the use of a community health approach in rural Africa. N Engl J Med. 2019 Jul 18; 381(3):219229.

31. Patel P, Ordunez P, DiPette D, Escobar MC, Hassell T, Wyss F et al. Improved blood pressure control to reduce cardiovascular disease morbidity and mortality: the Standardized Hypertension Treatment and Prevention Project. J Clin Hypertens (Greenwich). 2016 Dec; 18(12):1284-1294.

32. Schwartz JI, Guwatudde D, Nugent R, Kiiza CM. Looking at non-communicable diseases in Uganda through a local lens: an analysis using locally derived data. Global Health. 2014 Nov 19; 10:77.

33. Castelnuovo B, Kiragga A, Afayo V, Ncube M, Orama R, Magero S at al. Implementation of providerbased electronic medical records and improvement of the quality of data in a large HIV program in Sub-Saharan Africa. PLoS One. 2012; 7(12):e51631. 


\section{Tables}

\section{Table 1: Criteria used to assign ratings to the CFIR constructs that influence screening and treatment of HTN in the HIV clinics}

\begin{tabular}{|c|c|}
\hline Rating & Criteria \\
\hline-2 & $\begin{array}{l}\text { The construct has a negative influence to HTN screening and treatment in the HIV clinic. An } \\
\text { impeding influence in work processes, and/or an impeding influence in implementation efforts. The } \\
\text { majority of interviewees (at least two) described with explicit examples how the key or all aspects of } \\
\text { a construct manifests itself in a negative way. }\end{array}$ \\
\hline-1 & $\begin{array}{l}\text { The construct has a negative influence to HTN screening and treatment in the HIV clinic, an } \\
\text { impeding influence in work processes, and/or an impeding influence in implementation efforts. } \\
\text { Interviewees make general statements about the construct manifesting in a negative way but } \\
\text { without concrete examples: } \\
\text { - The construct is mentioned only in passing or at a high level without examples or evidence of } \\
\text { actual, concrete descriptions of how that construct manifests; } \\
\text { - There is a mixed effect of different aspects of the construct but with a general overall negative } \\
\text { effect; } \\
\text { - There is sufficient information to make an indirect inference about the generally negative } \\
\text { influence; and/or }\end{array}$ \\
\hline 0 & $\begin{array}{l}\text { A construct has neutral influence to HTN screening and treatment in the HIV clinic if: } \\
\text { - It appears to have neutral effect (purely descriptive) or is only mentioned generically without } \\
\text { valence; } \\
\text { - There is no evidence of positive or negative influence; } \\
\text { - Credible or reliable interviewees contradict each other } \\
\text { - There are positive and negative influences at different levels in the organization that balance } \\
\text { each other out; and/or different aspects of the construct have positive influence while others } \\
\text { have negative influence and overall, the effect is neutral. }\end{array}$ \\
\hline+1 & $\begin{array}{l}\text { The construct is a positive influence to HTN screening and treatment in the HIV clinic, a facilitating } \\
\text { influence in work processes, and/or a facilitating influence in implementation efforts. Interviewees } \\
\text { make general statements about the construct manifesting in a positive way but without concrete } \\
\text { examples: } \\
\text { - The construct is mentioned only in passing or at a high level without examples or evidence of } \\
\text { actual, concrete descriptions of how that construct manifests; } \\
\text { - There is a mixed effect of different aspects of the construct but with a general overall positive } \\
\text { effect; and/or } \\
\text { - There is sufficient information to make an indirect inference about the generally positive } \\
\text { influence. }\end{array}$ \\
\hline+2 & $\begin{array}{l}\text { The construct is a positive influence for HTN screening and treatment in the HIV clinic, a facilitating } \\
\text { influence in work processes, and/or a facilitating influence in implementation efforts. The majority } \\
\text { of interviewees (at least two) describe explicit examples of how the key or all aspects of a construct } \\
\text { manifests itself in a positive way. } \\
\text { Missing Interviewee(s) were not asked about the presence or influence of the construct; or if asked } \\
\text { about a construct, their responses did not correspond to the intended construct and were instead } \\
\text { coded to another construct. Interviewee(s) lack of knowledge about a construct does not necessarily } \\
\text { indicate missing data and may instead indicate the absence of the construct. }\end{array}$ \\
\hline
\end{tabular}

\section{Table 2: Number of participants involved in this study, by interview type and location}




\begin{tabular}{|l|r|r|r|r|r|}
\hline Data collection methods & $\begin{array}{l}\text { Mulanda } \\
\text { health center } \\
\text { IV }\end{array}$ & $\begin{array}{l}\text { Nagongera } \\
\text { health center } \\
\text { IV }\end{array}$ & $\begin{array}{l}\text { TASO } \\
\text { Tororo }\end{array}$ & $\begin{array}{l}\text { District Health } \\
\text { Team (DHT) }\end{array}$ & $\begin{array}{l}\text { Total } \\
\text { participants }\end{array}$ \\
\hline $\begin{array}{l}\text { Focus Group Discussion (FGDs) } \\
\text { for patients }\end{array}$ & 20 & 20 & 20 & 0 & 60 \\
\hline $\begin{array}{l}\text { In-depth Interviews (IDIs) for } \\
\text { patients }\end{array}$ & 4 & 4 & 4 & & 12 \\
\hline $\begin{array}{l}\text { Key Informant Interviews (KIIs) } \\
\text { for health care providers }\end{array}$ & 3 & 3 & 4 & & 11 \\
\hline Total number of participants & $\mathbf{2 7}$ & $\mathbf{2 7}$ & $\mathbf{2 8}$ & & $\mathbf{1}$ \\
\hline
\end{tabular}

\section{Figures}

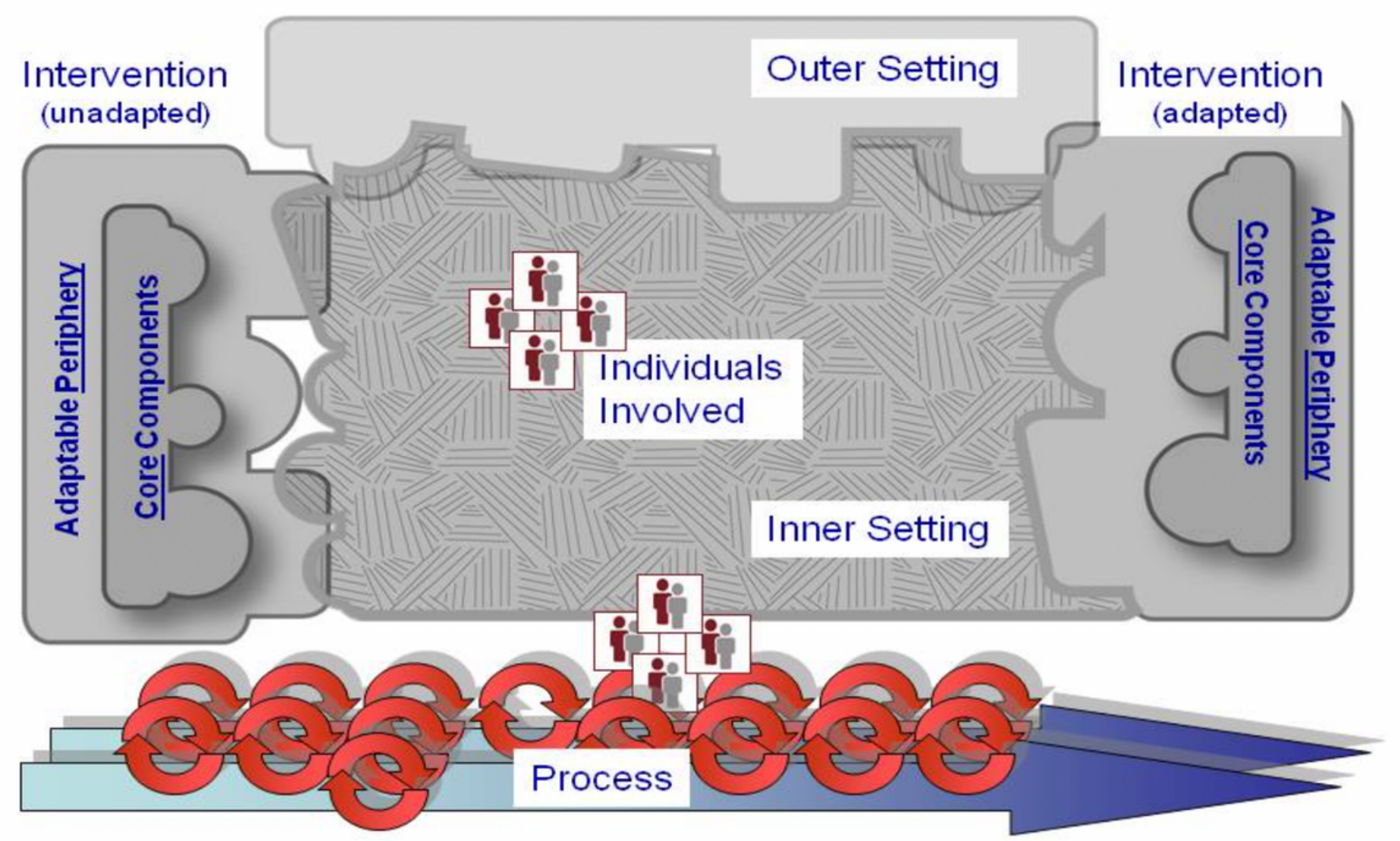

Figure 1

CFIR domains and their relationship 


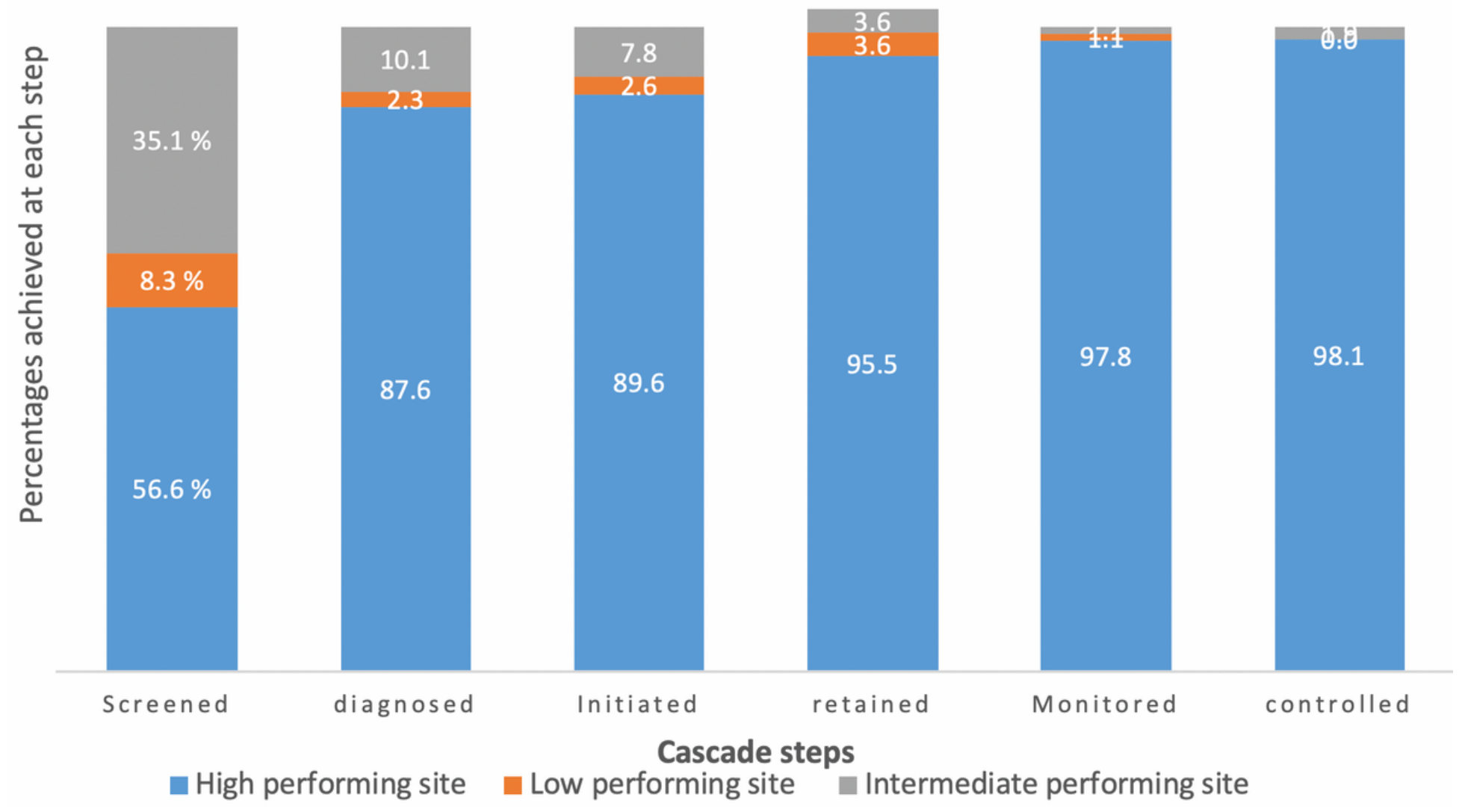

\section{Figure 2}

Differences in cascade outcomes across the three HIV clinics included in the study

\section{Supplementary Files}

This is a list of supplementary files associated with this preprint. Click to download.

- Additionalfiles.docx 